\title{
On Kinetic and Irreversible Thermodynamic Treatments of a Rarefied Gaseous Plasma Bounded by a Moving Plate
}

\author{
T. Z. Abdel Wahid ${ }^{1}$ and R. A. Shahein ${ }^{1-2}$
}

${ }^{1}$ Department of Mathematics and Computer Science, Faculty of Science, Menoufia University, Shebein El-Koom 32511, Egypt. 2 Department of Mathematics, Collage of Science, Taibah University, Al-Madinah Al-Munawarah, Saudi Arabia.

\section{ARTICLE INFO}

Received 28 December 2021
Accepted 04 February 2022

Keywords

Rarefied Gaseous plasma,

Fluid Mechanics,

Irreversible Thermodynamics,

Electron Velocity Distribution

function,

Travelling Wave Solution.

Correspondence

T. Z. Abdel Wahid

E-mail

taha_zakaraia@yahoo.com

\section{ABSTRACT}

The peculiarities of the Rayleigh problem (RP) governing equations of a rarefied gaseous plasma (RGP) are analyzed. They were proven to conform to the entropic performance for the RGP system using the moment method, separation of variables, associated with traveling-wave techniques in irreversible thermodynamics (IT) approach. Maxwell's equations and the Boltzmann equation (BE) of the BhatnagarGross- Krook (BGK) type were solved. The BE considerable advantage is that it allows us to analyze the depth performance of the equilibrium electrons' velocity distribution function (EVDF) and the perturbed EVDF and their implementation to determine how far the system is from the equilibrium state (ES). As a result, the contrast between the equilibrium EVDF and the perturbed EVDF was conceptually elucidated at various periods. This significant benefit enables us to consider our model's non-equilibrium IT properties. For this purpose, the derived EVDF should be employed in entropy, production, and other critical thermodynamic variables. After analyzing the results, we discovered that $\mathrm{H}$-theorem, thermodynamic principles, and Le Chatelier's law were consistent with our model. The Gibbs rule was used to express how the various influences of the forces acting on the system's internal energy modification (IEM) are expressed. The findings showed that the proposed model could accurately capture the performance. The suggested type could accurately predict RGP helium and argon gases performance in the upper atmosphere's ionized belts. 3D-Graphics representing the physical parameters were generated using analysis of variance calculations, and the results are thoroughly presented. The importance of this research stemmed from its broad array of utilization in micro-electro-mechanical systems (MEMS), physics, electrical engineering, and nano-electro-mechanical systems (NEMS) technologies in a variety of commercial and industrial utilization.

\section{Introduction}

The BE has several uses in MEMS \& NEMS technology. One of the most critical considerations driving the use of the BE in MEMS \& NEMS implementations is the knowledge that: Since their micron-scale size is generally comparable to the molecule mean free path under normal operating conditions. Thus, the Knudsen flow numbers in MEMS \& NEMS are generally far from the continuum regimes. Microflow is a term used to describe flow on a micron scale. The characteristic lengths of the flow gradient in microflows are usually modest and 
correspond to the molecules' mean free path. Often these MEMS \& NEMS typical lengths will be in the micron order or less, resulting in a Knudsen number between 0.001 to 10 . Resulting in fluid flows in the slip flow and transition flow conditions like most MEMS \& NEMS technology. Microflows in these regimes have characteristics that differ from typical flows with long characteristic lengths. The Navier-Stokes and other traditional continuum models cannot characterize and forecast micro and nano-flows. On the other hand, microflows impact the effectiveness of MEMS \& NEMS, such as micro pressure sensors, micro pumps, and valves ${ }^{[1]}$.

The problem of describing the motion of plasma was developed as a critical one. On the other hand, kinetic forms of the BE and macroscopic templates such as hydrodynamic type are extensively employed in plasma. While hydrodynamic systems are incredibly realistic to represent many observable occurrences, a fluid treatment is insufficient for some, like the RGP. A kinetic type must be applied to get out from the inadequacy of the hydrodynamic types in the RGP system. Nonetheless, kinetic type numerical simulations are prohibitively expensive in CPU time and memory capacity. To precisely explain the complex transfer phenomena in RGP streams circumstances, particle-based RGP dynamics should be used ${ }^{[1-3]}$. The transport field becomes complicated because nonequilibrium effects occur in the RGP flows [1]. Numerical solutions of the $B E$, which might be problematic, can characterize these non-equilibrium effects in kinetic gas theory. It is feasible to derive the equivalent macroscopic transport equations from a microscopic equation, such as the BE. The BE's classical approaches for deducing hydrodynamic-like equations are the Chapman-Enskog ${ }^{[4-6]}$ and Grad's moment methods [7-9]. In laboratory experiments and aerodynamics, the performance of the RGP in the presence of an infinite flat plate unexpectedly shifted in its plane, the RP, is of enormous interest. Particles collisions with rigid surfaces and particle binary collisions are predicted due to RGP rarefaction discontinuities in the surface's macroscopic parameters. Shedlovskii, El-Sakka, et al., Khater, et al. [9-11], among several others, investigated the RP for a great RGP using the collisionless $B E$, as well as the RGP 's dynamical and electromagnetic field (EMF) characteristics. This investigation aims to apply the precise traveling-wave techniques ${ }^{[12-16]}$ to solve the problem of the RP issue to estimate shear stress, velocity, viscosity coefficient, and induced magnetic and electric fields. IT performance of diamagnetic RGP must be studied by applying estimated EVDF to evaluate entropic predictions performance and associated IT functions.

\section{Geometry, Physical, and Mathematical Formulation}

Suppose that the top half of space $(y \geq 0)$, confined by an unlimited flat plate $(y=0)$, is complete with an RGP of electrons and ions Fig. 1. Because the ratio among electron and ion masses in ionized RGP is too slight $\left(\frac{m_{e}}{m_{i}} \ll 1\right)$, the ions will be treated as a motionless neutralizing background. Firstly, the RGP is incomplete ES and the wall rest. The plate then begins to move abruptly in its plane, with velocity $U_{0} e^{-\alpha t}$ is along the $x$ axis (as $U_{0}$ and $\alpha$ are constants). The temperature of the entire system (electrons + ions + surface) is maintained at a constant temperature. The nomenclature defines all physical parameters.

Where $\vec{F}$ operating upon every electron be calculated as follows ${ }^{[17-18]}$ :

$\vec{F}=-e \vec{E}+\frac{-e}{c_{o}}(\vec{c} \wedge \vec{B})$. By assuming

(1) $\vec{u} \equiv\left(V_{x}, 0,0\right), \vec{J} \equiv\left(q n V_{x}, 0,0\right), \vec{E} \equiv\left(E_{x}, 0,0\right)$ and $\vec{B} \equiv\left(0,0, B_{z}\right)$.

$E_{x}, B_{z}, J_{x}$, and $V_{x}$, are considered $(y, t)$ functions. Equations of Maxwell's are satisfied by this selection.

In the RGP, the EVDF is $f(y, \vec{c}, t)$, that can be calculated from the $B E$ [17-19] which can be composed in the BGK type ${ }^{[20-22]}$ as:

(2) $\frac{\partial f}{\partial t}+\vec{c} \cdot \frac{\partial f}{\partial \vec{r}}+\frac{\vec{F}}{m} \cdot \frac{\partial f}{\partial \vec{c}}=\frac{1}{\tau}\left(f_{0}-f\right)$, where $f_{0}=$ $n(2 \pi R T)^{-\frac{3}{2}} \exp \left(\frac{-(\vec{c}-\vec{u})^{2}}{2 R T}\right)$

By substitutions from (1) into (2), we get:

(3) $\frac{\partial f}{\partial t}+c_{y} \frac{\partial f}{\partial y}-\frac{e B_{z}}{m c_{0}}\left(c_{y} \frac{\partial f}{\partial c_{x}}-c_{x} \frac{\partial f}{\partial c_{y}}\right)+\frac{e E_{x}}{m} \frac{\partial f}{\partial c_{x}}=$ $\frac{1}{\tau}\left(f_{0}-f\right)$.

Considering the solution of Eq. (4) as in ${ }^{[7]}$ :

4)

$\begin{cases}f_{1}=n(2 \pi R T)^{-\frac{3}{2}}\left(1+\frac{c_{x} V_{x 1}}{R T}\right) \exp \left(\frac{-c^{2}}{2 R T}\right) & \text { for } c_{y}<0 \uparrow \\ f_{2}=n(2 \pi R T)^{-\frac{3}{2}}\left(1+\frac{c_{x} V_{x 2}}{R T}\right) \exp \left(\frac{-c^{2}}{2 R T}\right) & \text { for } c_{y}>0 \downarrow\end{cases}$

where $V_{x 1}$ and $V_{x 2}$ are two undetermined functions of variables $t$ and $\mathrm{y}$. 
Appling Gard's moment method ${ }^{[1,2]}$ multiplying Eq. (4) by $Q_{i}(\vec{c})$ and integrating overall values of $\vec{c}$, the kinetic transfer equations are obtained as:

$$
\text { (5) } \begin{aligned}
\frac{\partial}{\partial t} \int & Q_{i} f d \underline{c}+\frac{\partial}{\partial y} \int c_{y} Q_{i} f d \underline{c}+\frac{e E_{x}}{m} \int f \frac{\partial Q_{i}}{\partial c_{x}} d \underline{c}+ \\
+ & \frac{q B_{z}}{m c_{0}} \int\left(c_{x} \frac{\partial Q_{i}}{\partial c_{y}}-c_{y} \frac{\partial Q_{i}}{\partial c_{x}}\right) d \underline{c}=\frac{1}{\tau} \int\left(f_{0}-f\right) Q_{j} d \underline{c} .
\end{aligned}
$$

The integrals over velocity are computed using the formula ${ }^{[1,3]}$,

(6)

$$
\int Q_{i}(\vec{c}) f d \underline{c}=
$$

$\int_{-\infty}^{\infty} \int_{-\infty}^{0} \int_{-\infty}^{\infty} Q_{i} f_{1} d \underline{c}+\int_{-\infty}^{\infty} \int_{0}^{-\infty} \int_{-\infty}^{\infty} Q_{i} f_{2} d \underline{c}$, where $Q_{i}=Q_{i}(\vec{c}), i=1,2$ and $d \underline{c}=d c_{x} d c_{y} d c_{z}$

where $\quad c_{x}, c_{y}$ and $c_{z}$ correspondingly, three components of the electron speed along $x, y$, and $z$ axes. Moreover, the component of both $E$ and $B$ can be calculated from Maxwell's equations:

(7) $\frac{\partial E_{x}}{\partial y}-\frac{1}{c_{0}} \frac{\partial B_{z}}{\partial t}=0$

(8) $\frac{\partial B_{z}}{\partial y}-\frac{1}{c_{0}} \frac{\partial E_{x}}{\partial t}-\frac{4 \pi e n}{c_{0}} V_{x}=0$

where $\quad n=\int f d \underline{c}, \mathrm{nV}_{x}=\int c_{x} f d \underline{c}$, with the boundary and initial conditions

(9) $E_{x}(y, 0)=B_{z}(y, 0)=0$,

$E_{x}(y, t)$ and $B_{z}(y, t)$ are finite as $\mathrm{y} \rightarrow \infty$.

The dimensionless variables are introduced as,

10)

$$
\left.\begin{array}{l}
t=t^{\prime} \tau, y=\frac{y^{\prime} \tau}{\sqrt{2 \pi}} V_{T h}, V_{x}=V_{x}^{\prime} V_{T h}, \tau_{x y}=\tau_{x y}^{\prime} V_{T h}, M=\frac{c}{V_{T h}}, M_{p}=\frac{U_{0}}{V_{T h}} \\
B_{z}=B_{z}^{\prime} \frac{m c_{0} V_{T h}}{e \tau}\left(\frac{\sqrt{2 \pi}}{V_{T h}}\right), E_{x}^{\prime}=E_{x}^{\prime} \frac{m V_{T h}}{e \tau}, \rho=n m_{e}, V_{T h}=\sqrt{\frac{2 K T_{0}}{m_{e}}} .
\end{array}\right\}
$$

For $M^{2} \ll 1$ (small Mach number), we could consider that changes in temperature and density are negligible, i.e., $T=1+O\left(M^{2}\right)$ and $n=1+O\left(M^{2}\right)$. Let

(11) $V_{x}=\frac{1}{2}\left(V_{x 1}+V_{x 2}\right), \tau_{x y}=\frac{P_{x y}}{\rho U_{0} \sqrt{R T_{e} / 2 \pi}}=\left(V_{x 2}-\right.$ $\left.V_{x 1}\right)$.

By applying the non-dimension variable Eq. (5) for $Q_{1}=c_{x}$ and $Q_{2}=c_{x} c_{y}$ becomes

(12) $\frac{\partial V_{x}^{\prime}}{\partial t^{\prime}}+\frac{\partial \tau_{x y}^{\prime}}{\partial y^{\prime}}-E_{x}^{\prime}=0$,

(13) $\frac{\partial \tau_{x y}^{\prime}}{\partial t^{\prime}}+2 \pi \frac{\partial V_{x}^{\prime}}{\partial y^{\prime}}+\tau_{x y}^{\prime}=0$,

with the boundary and initial conditions

$$
\left.\begin{array}{c}
V_{x}^{\prime}\left(y^{\prime}, 0\right)=\tau_{x y}^{\prime}\left(y^{\prime}, 0\right)=0, \\
2 V_{x}^{\prime}\left(0, t^{\prime}\right)+\tau_{x y}^{\prime}\left(0, t^{\prime}\right)=2 M_{p} e^{-\alpha_{1} t^{\prime}} \\
V_{x}^{\prime} \text { and } \tau_{x y}^{\prime} \text { are finite as } \mathrm{y} \rightarrow \infty, \alpha_{1}=\alpha \tau .
\end{array}\right\}
$$

In equations (7)-(9) and (12)- (14), we remove the dash over the dimensionless variables for brevity's purpose. Thus, we have the following equations system (disregard current of displacement) ${ }^{[23]}$ :

(15) $\frac{\partial V_{x}}{\partial t}+\frac{\partial \tau_{x y}}{\partial y}-E_{x}=0$,

(16) $\frac{\partial \tau_{x y}}{\partial t}+2 \pi \frac{\partial V_{x}}{\partial y}+\tau_{x y}=0$,

(17) $\frac{\partial E_{x}}{\partial y}-\frac{\partial B_{z}}{\partial t}=0$,

(18) $\frac{\partial B_{z}}{\partial y}-\alpha_{0} V_{x}=0, \quad$ where $\alpha_{0}=\frac{V_{T h}{ }^{2} \tau^{2} e^{2} n_{e}}{m_{e} c_{0}^{2}}$,

with the boundary and initial conditions

(19)

$$
\begin{gathered}
V_{x}(y, 0)=\tau_{x y}(y, 0)=E_{x}(y, 0)=B_{z}(y, 0)=0, \\
2 V_{x}(0, t)+\tau_{x y}(0, t)=2 M_{p} e^{-\alpha_{1} t}, \text { for } t>0 ; \\
V_{x}, \tau_{x y}, E_{x} \text { and } B_{z} \text { are finite as } \mathrm{y} \rightarrow \infty .
\end{gathered}
$$

We can reduce our basic Eqs. (15-18), to one equation:

(20) $\frac{\partial^{4} V_{x}(y, t)}{\partial t^{2} \partial y^{2}}-\alpha \frac{\partial^{2} V_{x}(y, t)}{\partial t^{2}}-\frac{\partial^{3} V_{x}(y, t)}{\partial t \partial y^{2}}-\alpha \frac{\partial V_{x}(y, t)}{\partial t}-$ $2 \pi \frac{\partial^{4} V_{x}(y, t)}{\partial t^{4}}=0$.

\section{Solution of the Problem}

We will apply traveling-wave techniques ${ }^{[12-16]}$ since the traveling wave variable

(21) $\xi=-y-M t$, we change the partial derivatives to ordinary derivatives, where

(22) $\frac{\partial}{\partial t}=-M \frac{d}{d \xi}, \frac{\partial}{\partial y}=-\frac{d}{d \xi}$ and $\frac{\partial^{a}}{\partial t^{a}}=(-1)^{a} M^{a} \frac{d^{a}}{d \xi^{a}}$, $\frac{\partial^{a}}{\partial y^{a}}=(-1)^{n} \frac{d^{a}}{d \xi^{a}}$ as $\boldsymbol{a}$ is a +ve integer.

Substituting Eqs. (21-22) into Eq. (20), we have:

$$
\left(M^{2}-2 \pi\right) \frac{d^{4} V_{x}(\xi)}{d \xi^{4}}+M \frac{d^{3} V_{x}(\xi)}{d \xi^{3}}-\alpha M^{2} \frac{d^{2} V_{x}(\xi)}{d \xi^{2}}+
$$

$\alpha M \frac{d V_{x}(\xi)}{d \xi}=0$

Integrating Eq. (23) concerning $\xi$ once, we obtain:

$$
\left(M^{2}-2 \pi\right) \frac{d^{3} V_{x}(\xi)}{d \xi^{3}}+M \frac{d^{2} V_{x}(\xi)}{d \xi^{2}}-\alpha M^{2} \frac{d V_{x}(\xi)}{d \xi}+
$$

$\alpha M V_{x}(\xi)=C_{1}$,

where $C_{1}$ is the integration constant, the boundary and initial conditions become:

$(25-A) \quad E_{x}(\xi=0)=B_{z}(\xi=0)=\tau_{x y}(\xi=0)=0$,

$\left.(25-B) 2 V_{x}(\xi=-M)+\tau_{x y}(\xi=-M)=2 M_{p} e^{-\alpha_{1}}\right\}$

$(25-C) \quad V_{x}, \tau_{x y}, E_{x}$ and $B_{z}$ are finite as $\xi \rightarrow-\infty$.

By solving the ordinary differential Eq. (24), we obtain the form of the general solution:

(26) $V_{x}(\xi)=C_{2} e^{\theta_{1} \xi}+C_{3} e^{\theta_{2} \xi}+C_{4} e^{\theta_{3} \xi}+\frac{C_{1}}{M \alpha}$ 


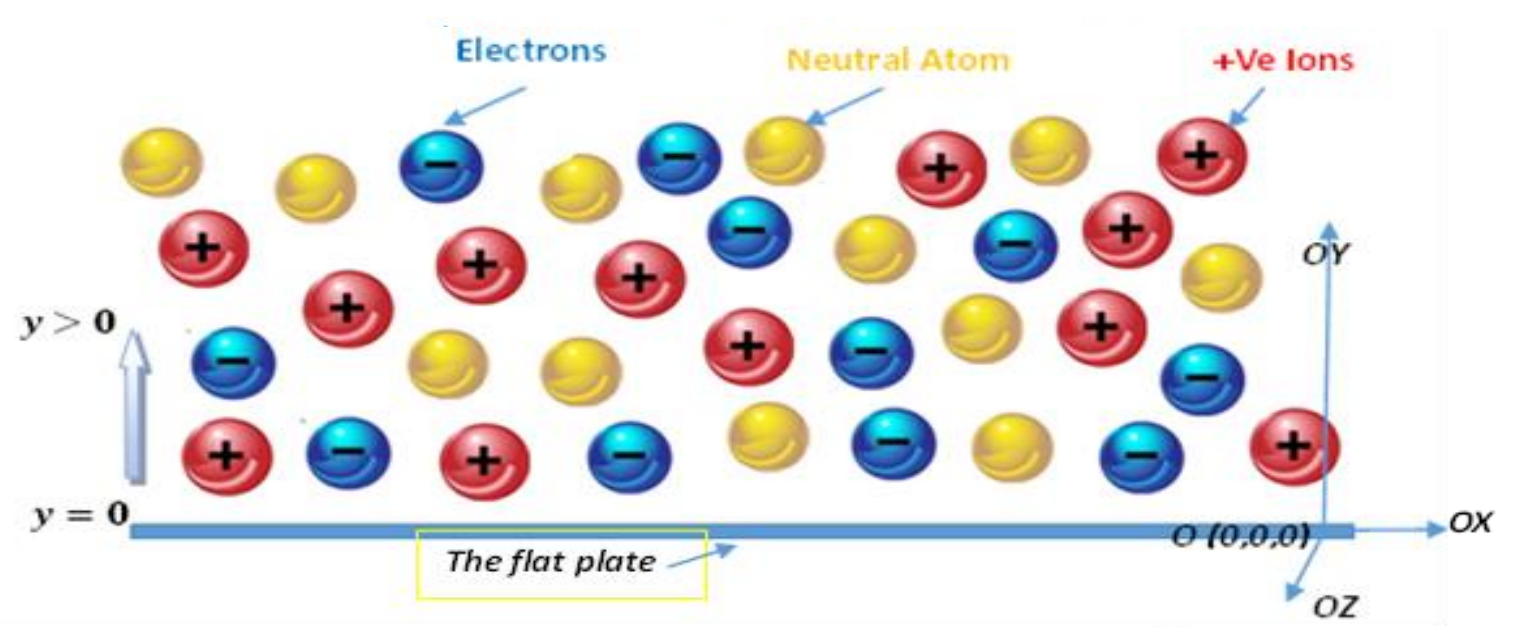

Fig. 1 Schematic representation of our problem. The rigid flat plate located at $y=0$ and the plasma components occupied the half-space $y>0$

where $C_{2}, C_{3}$ and $C_{4}$ are integrating constants calculated from the boundary and initial conditions $\theta_{1}, \theta_{2}$ and $\theta_{3}$ are the three roots of the algebraic auxiliary equation: $\left(M^{2}-2 \pi\right) \theta^{3}+M \theta^{2}-\alpha M^{2} \theta+$ $\alpha M=0$.

Substituting from Eqs. (21-22) into Eqs. (15-18) we obtain:

(27) $-M \frac{\partial V_{x}}{\partial \xi}-\frac{\partial \tau_{x y}}{\partial \xi}-E_{x}=0$,

(28) $-M \frac{\partial \tau_{x y}}{\partial \xi}-2 \pi \frac{\partial V_{x}}{\partial \xi}+\tau_{x y}=0$,

(29) $-\frac{\partial E_{x}}{\partial \xi}+M \frac{\partial B_{z}}{\partial \xi}=0$,

(30) $-\frac{\partial B_{z}}{\partial \xi}-\alpha_{0} V_{x}=0$,

Integrating Eq. (30) concerning $\xi$, we get:

(31) $B_{z}(\xi)=-\alpha_{0} \int V_{x}(\xi) d \xi$

Substituting from Eq. (26) into Eq. (31), we obtain:

$$
B_{z}(\xi)=-\alpha_{0}\left(\frac{C_{2}}{\theta_{1}} e^{\theta_{1} \xi}+\frac{C_{3}}{\theta_{2}} e^{\theta_{2} \xi}+\frac{C_{4}}{\theta_{3}} e^{\theta_{3} \xi}+\right.
$$

$\left.\frac{C_{1} \xi}{M^{2} \alpha}\right)+C_{5}$

The condition (25-C) implies that $C_{1}=$ zero, thus

(33) $B_{z}(\xi)=-\alpha_{0}\left(\frac{C_{2}}{\theta_{1}} e^{\theta_{1} \xi}+\frac{C_{3}}{\theta_{2}} e^{\theta_{2} \xi}+\frac{C_{4}}{\theta_{3}} e^{\theta_{3} \xi}\right)+C_{5}$.

Integrating Eq. (29) concerning $\xi$ then we get:

(34) $E_{x}(\xi)=M B_{z}(\xi)+C_{6}$.

Substituting from Eqs. (33) into Eq. (34), we obtain:

$$
E_{x}(\xi)=-\alpha_{0} M\left(\frac{C_{2}}{\theta_{1}} e^{\theta_{1} \xi}+\frac{C_{3}}{\theta_{2}} e^{\theta_{2} \xi}+\frac{C_{4}}{\theta_{3}} e^{\theta_{3} \xi}\right)+
$$

$C_{5} M+C_{6}$,

Integrating Eq. (27) concerning $\xi$ then we get:

(36) $\tau_{x y}(\xi)=-M V_{x}(\xi)-\int E_{x}(\xi) d \xi$

Substituting from Eqs. $(26,37)$ into Eq. (38), we obtain:
(37) $\tau_{x y}(\xi)=-M\left(C_{2} e^{\theta_{1} \xi}+C_{3} e^{\theta_{2} \xi}+C_{4} e^{\theta_{3} \xi}\right)+$ $\alpha_{0} M\left(\frac{C_{2}}{\theta_{1}{ }^{2}} e^{\theta_{1} \xi}+\frac{C_{3}}{{\theta_{2}}^{2}} e^{\theta_{2} \xi}+\frac{C_{4}}{{\theta_{3}}^{2}} e^{\theta_{3} \xi}\right)+C_{5} M \xi+C_{6} \xi+$ $C_{7}$ applying Eq. (28) together with condition (25-C), we obtain that $C_{5}=\mathrm{C}_{6}=\mathrm{C}_{7}=0$ while the three constants $C_{2}, C_{3}$ and $C_{4}$ can be calculated from the conditions (25), which gives the three following equations:

$$
E_{x}(\xi=0)=0, \tau_{x y}(\xi=0)=0,2 V_{x}(\xi=
$$$$
-M)+\tau_{x y}(\xi=-M)=2 M_{p} e^{-\alpha_{1}}
$$

Substituting from Eqs. $(26,35,37)$ into the system of Eqs. (38), we obtain three algebraic equations in three unknown constants $C_{2}, C_{3}$ and $C_{4}$, which can be solved easily by usual methods of algebra to get the values of $C_{2}, C_{3}$ and $C_{4}$ to obtain the complete solution of the problem, as we will do in the applied example.

Substituting the calculated velocities $V_{x 1}$ and $V_{x 2}$ from Eqs. $(11,26,37)$ into Eq. (4) for $f_{1}$ and $f_{2}$ we may now begin to investigate the system's non-equilibrium thermodynamic characteristics.

\section{The Non-Equilibrium Thermodynamic Descriptions}

The IT processes are still representing a hot exciting topic. That is due to the theory's broad theoretical significance and diverse applicability in various fields. We begin by calculating the entropy per unit mass $S$, going back to the basics of the $\mathrm{H}$-theorem. It has written as ${ }^{[24-26]}$ :

(39) $S=-\int f \ln f d \underline{c}=-\left(\int f_{1} \ln f_{1} d \underline{c}+\right.$ $\left.\int f_{2} \ln f_{2} d \underline{c}\right)=-\pi^{\frac{3}{2}}\left[\left(V_{x 1}{ }^{2}+V_{x 2}^{2}\right)-\frac{3}{2}\right]$ 
Moreover, we get entropy flow in the y-direction:

(40) $J_{y}{ }^{(S)}=-\int c_{y} f \ln f d \underline{c}=-\left(\int c_{y} f_{1} \ln f_{1} d \underline{c}+\right.$ $\left.\int c_{y} f_{2} \ln f_{2} d \underline{c}\right)=\left[\pi\left(V_{x 1}{ }^{2}+V_{x 2}{ }^{2}\right)\right]$

In its global form, the principle of entropy generation [28-30] is expressed as:

(41) $\sigma=\frac{\partial S}{\partial t}+\vec{\nabla} \cdot \overrightarrow{J^{(S)}}$.

We may calculate the thermodynamic force equivalent to the control factors $M_{p}{ }^{[9]}$ using the grand principle of IT:

(42) $X_{M p}=\frac{\partial S}{\partial M_{p}}$.

The connection among entropy generation and thermodynamic forces, but from the other side, takes the form ${ }^{[24-26]}$ :

(43) $\sigma=\sum_{i} \sum_{j} L_{i j} X_{i} X_{j}=L_{11} X_{M p}{ }^{2}$.

In our problem, the condition of the Onsager inequality will be satisfied by the kinetic coefficient $L_{11}$ and the thermodynamic force $X_{M p}$ as $L_{11} \geq 0$.

To investigate the system's IEM. The extended Gibbs relation ${ }^{[9,27]}$ is introduced, consisting of EMF energy as a part of the total system energy. We distinguish paramagnetic and diamagnetic RGP [9].

Case1: The RGP is paramagnetic, the IEM is stated in terms of the extended thermodynamics coordinates $S$, $P$, and $m$, which correspond to the intensive thermodynamics' coordinates $T, E$, and $B$, correspondingly, the different influences in the IEM in Gibbs methodology:

(34) $d U=d U_{S}+d U_{p o l}+d U_{\text {para }}$, where

$d U_{S}=T d S$ is the IEM because of a variant of the entropy,

$d U_{p o l}=E d P$ is the IEM because of a variant of polarization, and

$d U_{\text {para }}=B d m$ is the IEM because of the variant of magnetization. Here $m$ is calculated from the Equation [9, 27]: $\frac{\partial S}{\partial m}=-\frac{B}{T} \Rightarrow m=-\int\left(\frac{T}{B} \frac{\partial S}{\partial y}\right)_{t} d y$. presenting the nondimensional quantities $U^{\prime}=\frac{U}{K T_{0}}, m^{\prime}=$ $m\left(\frac{1}{e \tau V_{T h}}\right), p^{\prime}=p\left(\frac{1}{e \tau V_{T h}}\right)$ in the Gibbs' rule to get (dropping primes): $d U=d S+\varepsilon_{1} E d p+\varepsilon_{1} B d m$.

Case 2: If the RGP is diamagnetic, the IEM because of the extensive $S, P$, and $B$ and intensive variables $T, E$, and $m$, correspondingly. Therefore, there are three influences in the IEM in the Gibbs rule given by: $d U=$ $d U_{S}+d U_{p o l}+d U_{d i a}$, where $d U_{\text {dia }}=-m d B$ is the IEM because of induced magnetic induction variant $m=$ $T \frac{\partial S}{\partial B}{ }^{[11,31]}$. As a result, the nondimensional form of $d U$ in just this example is:

$$
\begin{aligned}
& d U=d S+\varepsilon_{1} E d p-\varepsilon_{1} m d B, \text { where } \\
& \varepsilon_{1}=\left(\frac{m_{e} V_{T h}{ }^{2}}{K T_{0}}\right) \quad, d S=\left(\frac{\partial S}{\partial r}\right) \delta y+\left(\frac{\partial S}{\partial t}\right) \delta t ; \delta y= \\
& 3, \delta t=20 .
\end{aligned}
$$

\section{Discussion}

The unsteady performance of RGP is investigated by applying the traveling-wave techniques and the kinetic theory of IT processes. Our calculations are based on detailed data for RGP in Argon RGP ${ }^{[28]}$ as a diamagnetic medium in which the Argon RGP loses electron pairs as a function of the voltage used to ionize the Argon atoms, exposed to the following parameters:

$K_{B}=1.3807 \times 10^{-16} \mathrm{erg} / \mathrm{K}^{o}, T_{0}=300 \mathrm{~K}^{o}, n_{e}=$ $7 \times 10^{14} \mathrm{~cm}^{-3}, d=3.84 \times 10^{-8} \mathrm{~cm}$ (Argon atom diameter), the electron mass, charge $m_{e}=$ $9.093 \times 10^{-28} \mathrm{gm}, e=4.8 \times 10^{-10} \mathrm{esu}$ are applied to calculate the dimensionless parameter $\alpha_{0}=9.37$, the particles Mach number $M=10^{-1}$ and the mean free path of the RGP $\lambda=\frac{1}{\sqrt{2} \pi n_{e} d^{2}}=$ $0.2180 \mathrm{~cm}$ that comparing with Debye's length $\lambda_{D e}=\sqrt{\frac{K_{B} T_{0}}{4 \pi n_{e} e^{2}}}=6.389 \times 10^{-6} \mathrm{~cm}, \varepsilon_{1}=1.667$, and the plate Mach number $M_{p}=5 \times 10^{-2}$.

Fig. 2 sheds light upon a comparison of the combination perturbation EVDF $f\left[f_{1}\right.$ (green), $f_{2}$ (red) $]$ with the equilibrium EVDF $f_{0}($ Yellow - Grid $)$ at various time values (a) as $t=1$, (b) as $t=30$, (c) as $t=40$, and (d) as $t=70$ all figures done for a fixed $(y=0.3)$ with plate's Mach number $M a=0.05$. It shows that the distance from ES is small. The system, over time, works to restore the ES, which proves that the system complies with the $2^{\text {nd }}$ law of thermodynamics and conforms to the Le Chatelier ES principle. Fig. 3 Illustrates (a) the equilibria EVDF $f_{0}$ (b) the combination perturbation EVDF $f$, and (c) a combination of equilibrium and non-equilibrium EVDF. All of them show that the system almost approaches ES as $t=100$. The advantage of the EVDF representation and calculation is that it illustrates how the system is far from ES and when it may reach an ES. We can do that after many evolutions of both perturbed and 
equilibrium EVDF and compare them every time. When the equilibrium EVDF matches the unbalanced EVDF thus, we conclude that the system reaches the
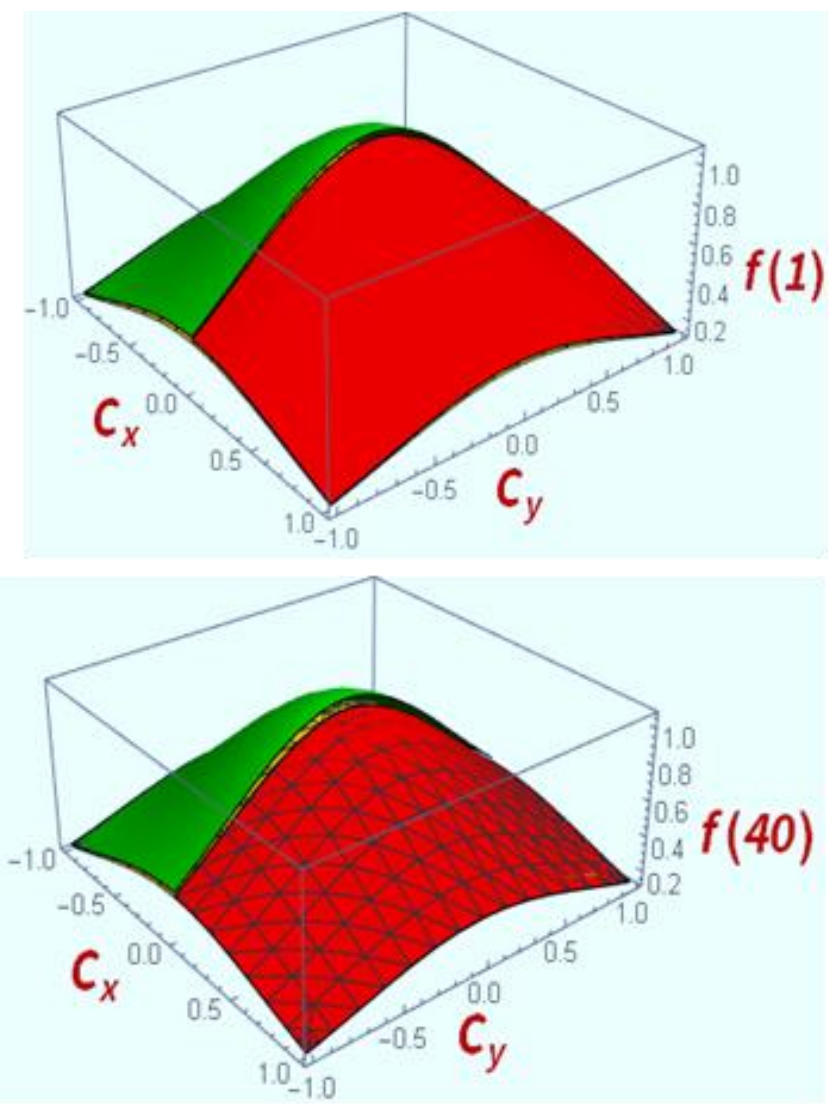

ES. That advantage cannot be reached by solving macroscopic types like Navier-Stocks and other macroscopic magnetohydrodynamic models.
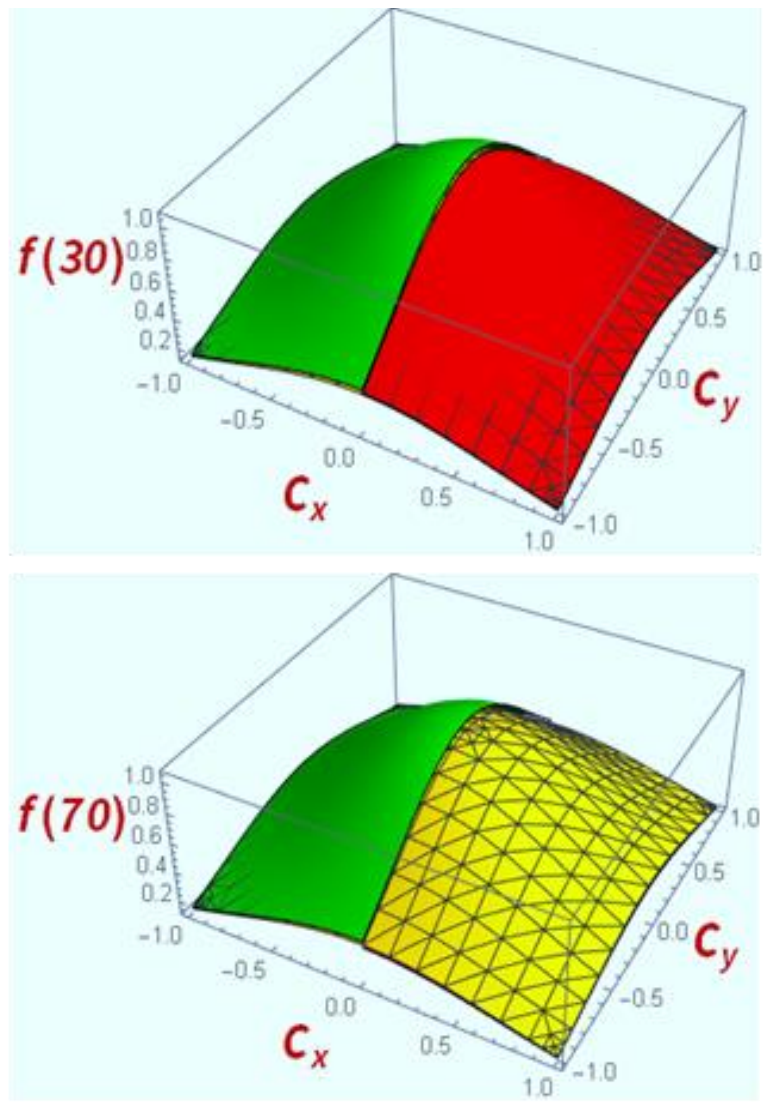

Fig. 2 Comparison of the combination perturbation EVDF $f\left[f_{1}\right.$ (Green), $f_{2}$ (Red)] with the equilibria EVDF $f_{0}$ (Yellow-Grid) at $(t=1,30,40$ and 70$)$ for a fixed $(y=0.3)$ with plate's Mach number $M a=0.05$
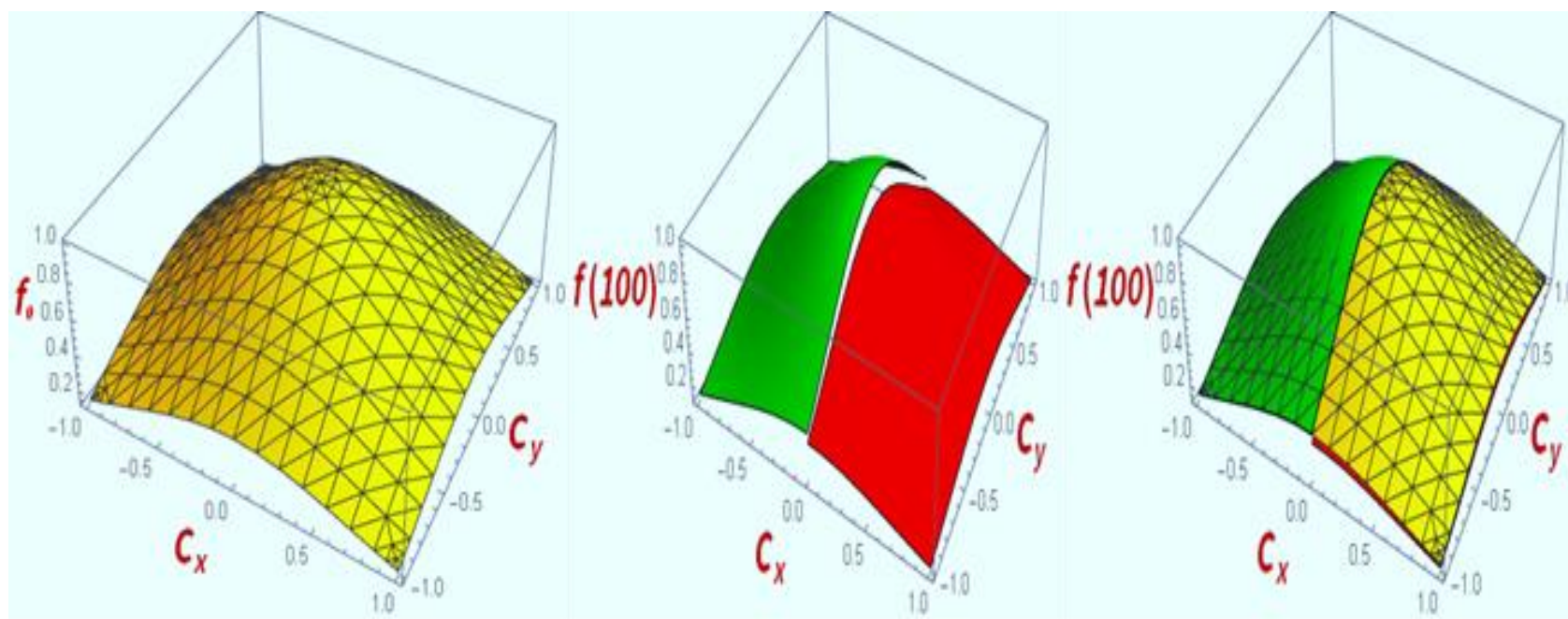

Fig. 3 Distinction of the equilibria EVDF $f_{0}$ (Yellow-Grid) and the coupled perturbed EVDF $f\left[f_{1}\left(\right.\right.$ Green), $f_{2}($ Red $\left.)\right]$ and at $(\mathrm{t}=$ 100) for a fixed $(y=0.3)$ with plate's Mach number $M a=0.05$ 
The mean velocity is seen from Fig. 4, at the neighborhood of the sudden movement of the plate, it is a maximum $\left(\approx M_{p}\right)$ and then declines time exponentially. It decreases non-linearly with the distance $y$.

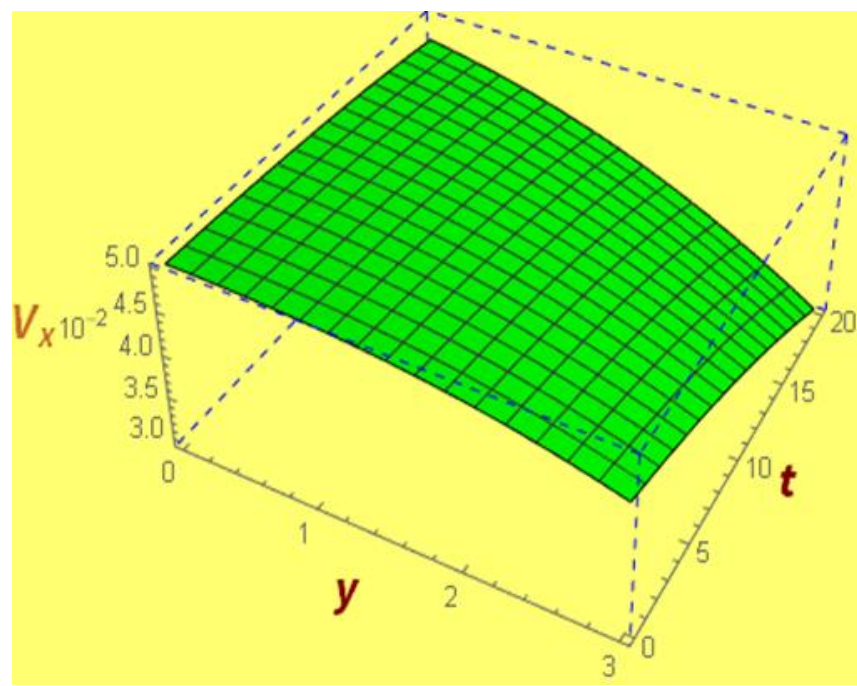

Fig. 4 The velocity $V_{x}$ vs. $y$ and $\mathrm{t}$

The mean velocity is seen from Fig. 4, at the neighborhood of the sudden movement of the plate, it is a maximum $(\approx$ $M_{p}$ ) and then declines time exponentially. It decreases nonlinearly with the distance $y$. A similar performance holds for the shear stress $\tau_{x y}$ except that $\tau_{x y}$ equals zero at $\mathrm{t}=0$ and $y=0$ also, it has a negative sign owing to its direction from bottom to upper, see Fig. 5 .

We considered the RGP is taken as Newtonian ${ }^{[9]}$. The viscosity impairs the motion, which is non-linear incrementally increasing as the RGP deviates from the surface. It increases with increasing time because of the decrement of the velocity of the electrons, see Figs. 6, 7 .

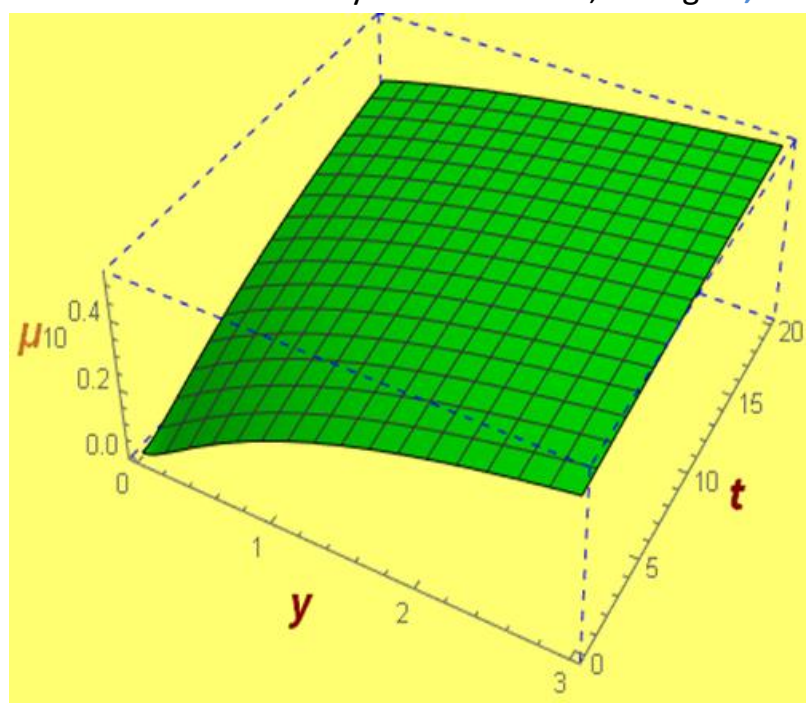

Fig. 6 Viscosity coefficient $\mu$ vs. $y$ and $\mathrm{t}$
A similar performance holds for the shear stress $\tau_{x y}$ except that $\tau_{x y}$ equals zero at $\mathrm{t}=0$ and $y=0$ also, it has a negative sign owing to its direction from bottom to upper, see Fig. 5.

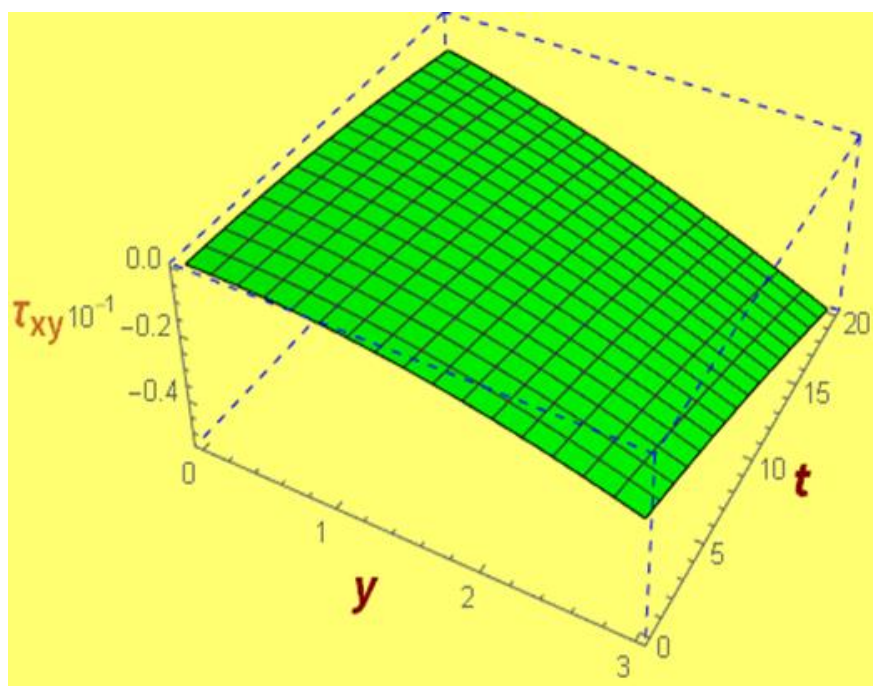

Fig. 5 The shear stress $\tau_{x y}$ vs. $y$ and $\mathrm{t}$

The amplitude of the induced electric field increases exponentially with time $t$ and distance $y$; see Fig. 7 . The same observations hold for the induced magnetic; see Fig. 8.

The thermodynamic performance is illustrated in Figs. (9-15). The performance of the entropy $\boldsymbol{S}$ has a good agreement with the $2^{\text {ed }}$ law of thermodynamic and the $\mathrm{H}$-theorem that it increases with the increment of time t, see Fig. 9. Also, Fig. 10 verifies how the performance of the system coincides with $\mathrm{H}$ theorem since the entropy generation is positive $\sigma \geq 0$ [24-26]. Indeed, the coefficient $L_{M p}$ satisfies the thermodynamics restrictions as it has a non-negative value for all the interval time and all y; see Fig. 11, 12.

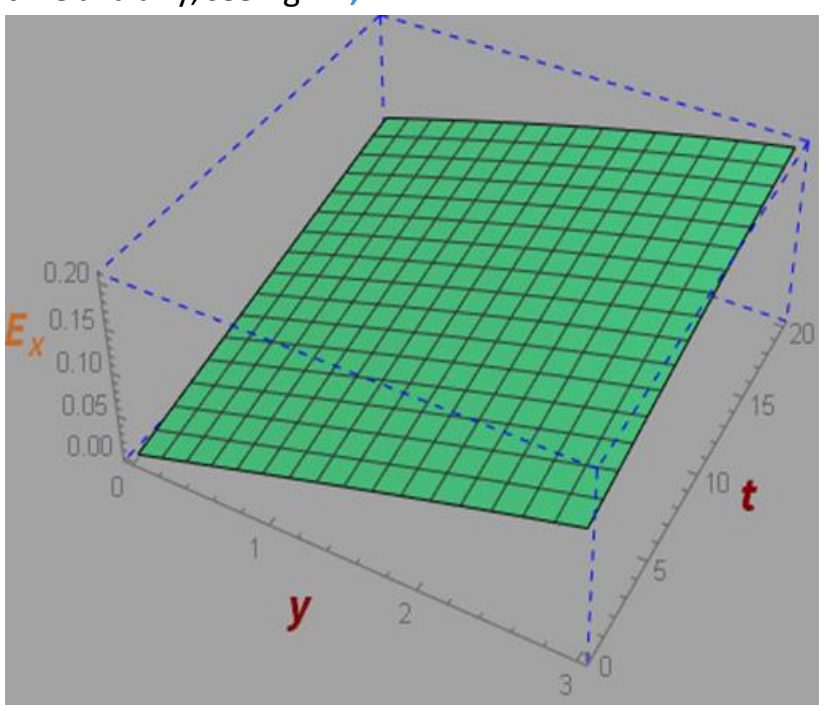

Fig. 7 The electric field $E_{x}$ vs. $y$ and $t$ 


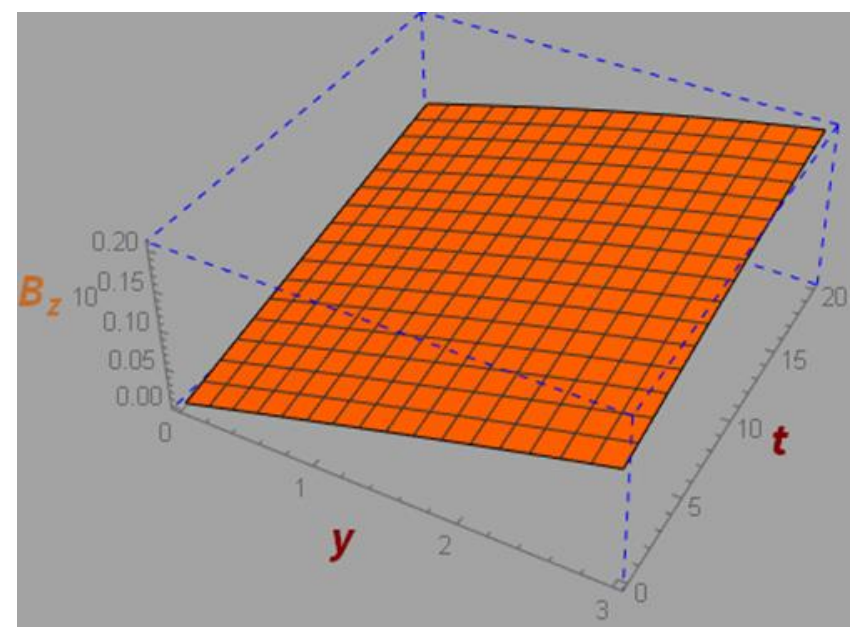

Fig. 8 The magnetic field $B_{z}$ vs. $y$ and $t$

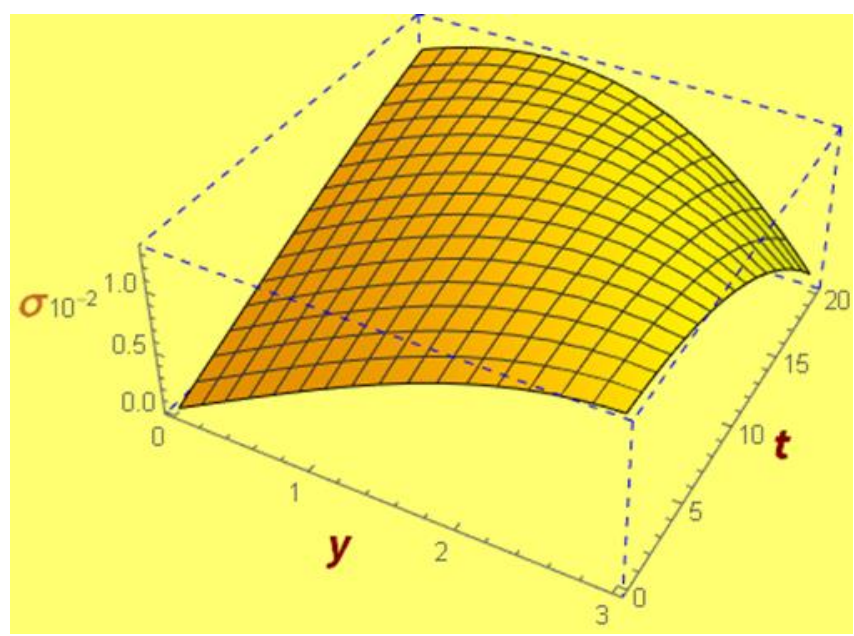

Fig. 10 Entropy production $\sigma$ vs. $y$ and $t$

The electron loses (or gains) energy as it passes through RGP due to interactions with the particles of the surroundings caused by RGP polarization and collisions. The forces acting on electrons in the RGP from the EMF generated by the particles affect an electron's energy loss (or gain). The abruptly shifting surface produces work on the RGP, altering the IEM of the RGP ${ }^{[29]}$.

As shown in Fig. 13, IEM is smoothly damping with time away from the plate due to entropy variant $d U_{S}$. It also has a linear rise in moving plate proximity owing to the energy wasted and received from the particles and surface.

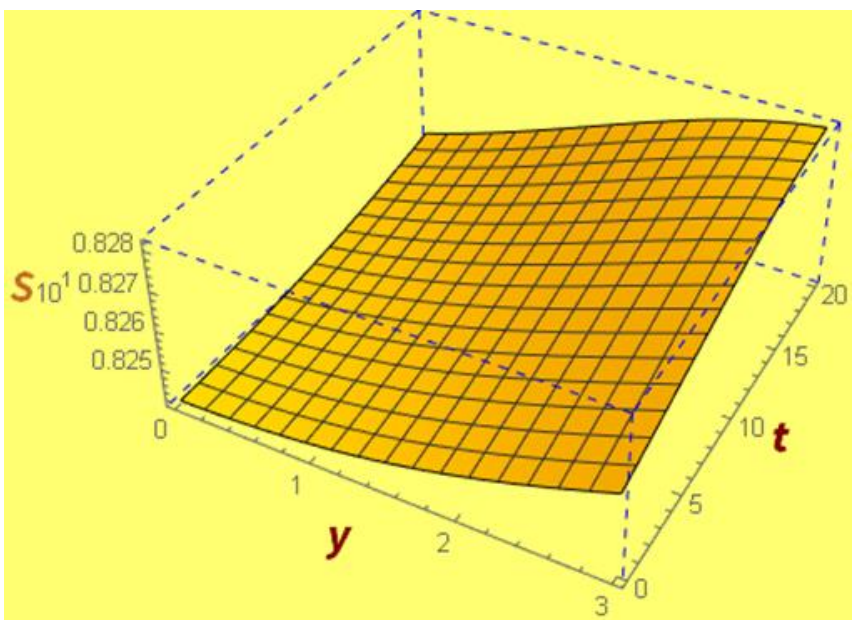

Fig. 9 Entropy $S$ vs. y and t

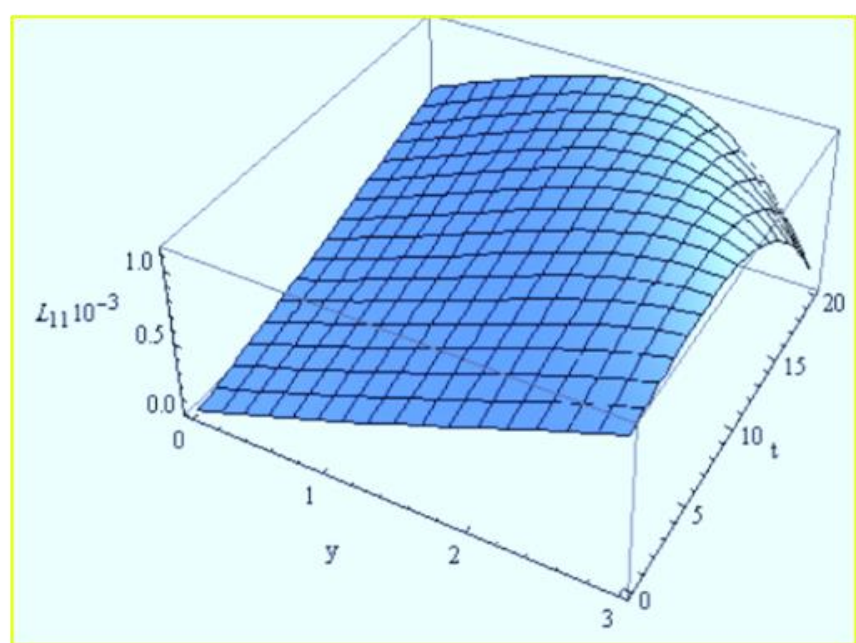

Fig. 11 The kinetic coefficient $L_{M p}$ vs. y and t

Fig. 14 demonstrates that the internal energy owing to the polarization variant grows as both the time $t$ and the distance $y$ increase, which is consistent with the performance of EMFs (see Figs. 7,8,14).

While the IEM varies smoothly between two maximum negative values for the diamagnetic RGP, we found $d U_{d i a}$ variants in the intensity of the induced magnetic field, as seen in Fig. 15.

Because of variations in the strength of the EMF, the IEM ranges non-linearly between two maximum negative values for the Para-magnetic $R G P, d U_{p a r}$, As shown in Fig. 16. 


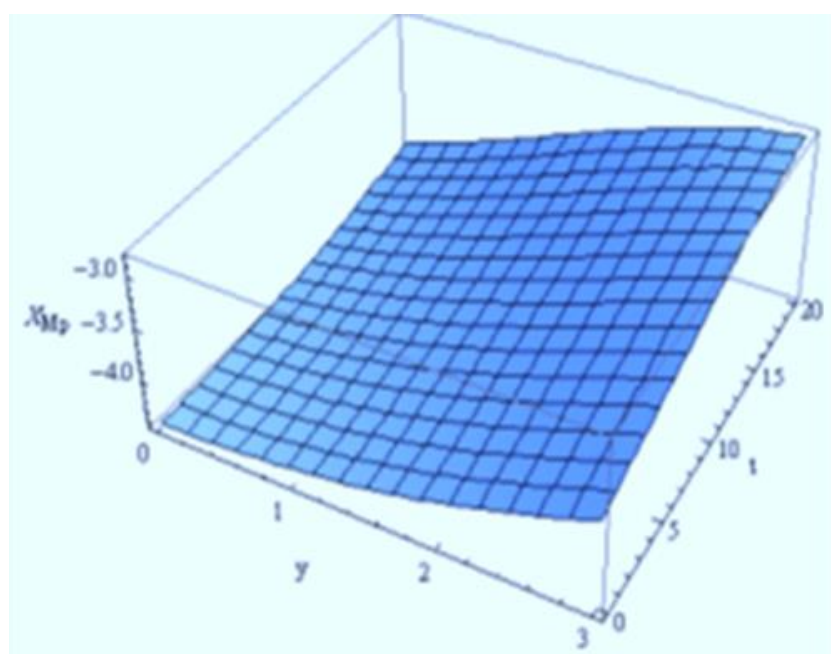

Fig. 12 Thermodynamic force $X_{M p}$ vs. $y$ and $t$

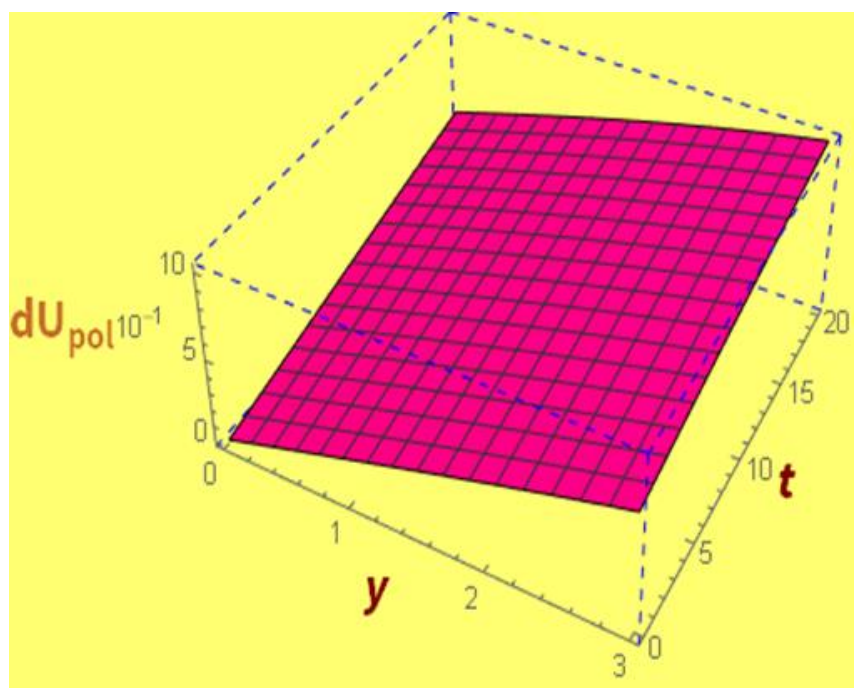

Fig. $14 d U_{\text {pol }}$ vs. $y$ and t

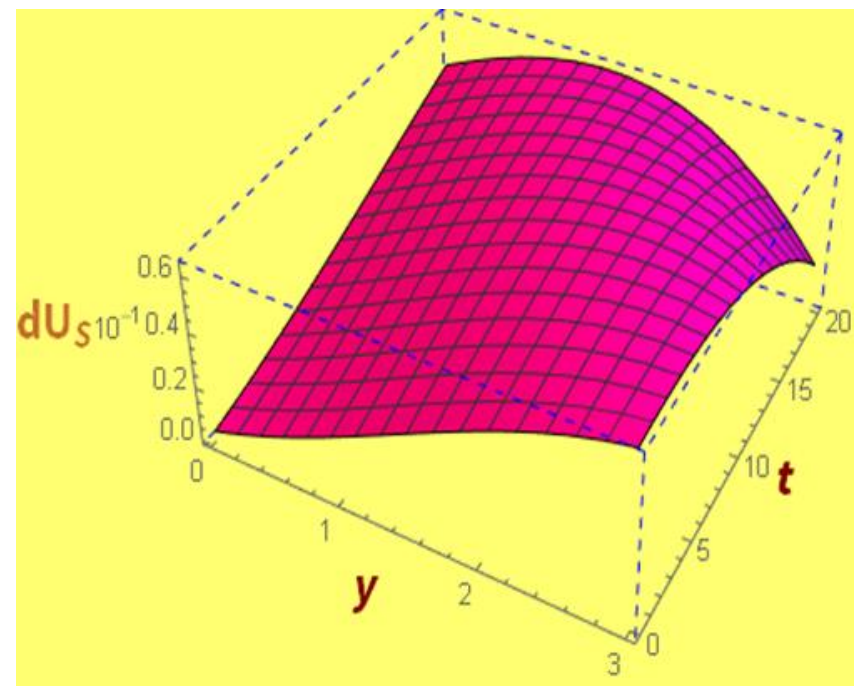

Fig. $13 d U_{s}$ vs. y and t

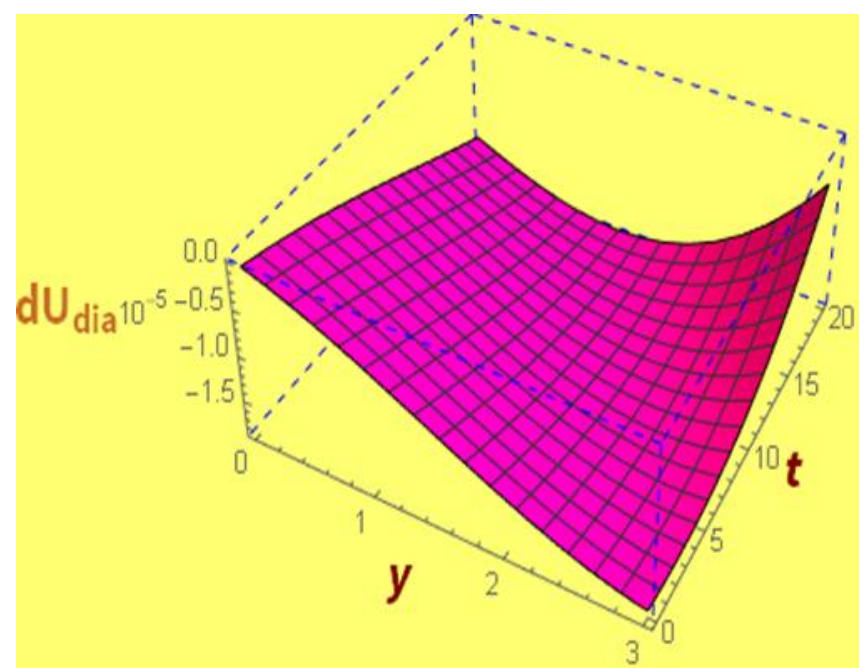

Fig. $15 \mathrm{dU}_{\mathrm{dia}}$ vs. $\mathrm{y}$ and $\mathrm{t}$

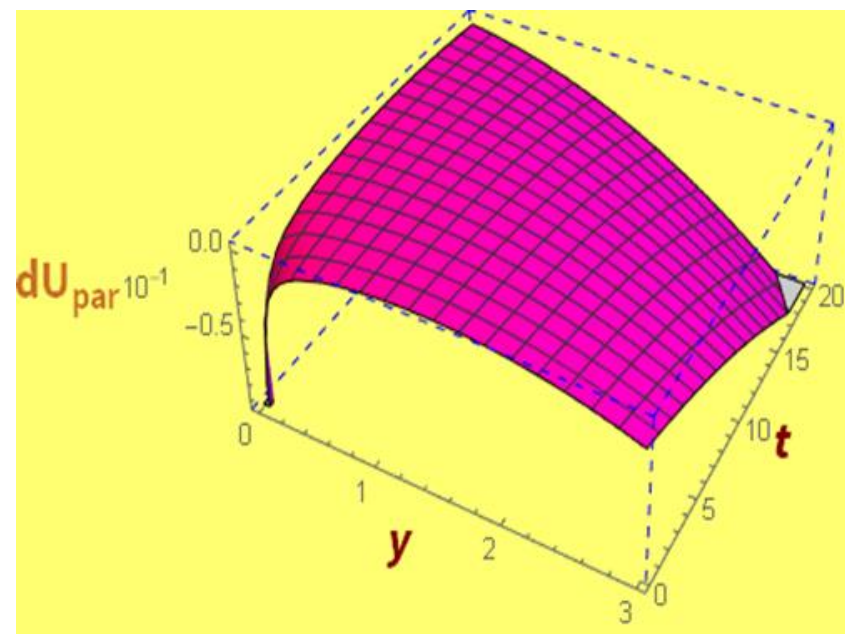

Fig. $16 d U_{d i a}$ vs. $y$ and $t$ 


\section{Conclusions}

The finding of the investigation of the unsteady BGK type of the BE in the case of an RGP applying the moments, traveling wave, and separation of variables methods of the two-sided EVDF and Maxwell's equations is developed within the restrictions of slight deviation from ES, RGP, and low Mach numbers. This technique allows us to calculate the flow velocity and other plasma parameters and variables. We evaluated the entropy, its production, and other important thermodynamic important variables by utilizing them in the EVDF and applying the $\mathrm{H}$-theorem. The results correlated well with the $\mathrm{H}$-theorem, thermodynamics rules, and Le Chatelier's concept. Via Gibbs' formula, the ratios between the various influences of the IEM are evaluated.

We found that the maximum numerical values of the three IEM influences in the Dia-magnetic case are ordered in magnitude as:

$$
d U_{S}: d U_{\text {pol }}: d U_{\text {dia }}=1: 1.67 \times 10: 2.5 \times 10^{-4} \text {. }
$$

While In the Para-magnetic scenario, the maximum quantities of the three IEM impacts are ordered in magnitude as:

$$
d U_{S}: d U_{p o l}: d U_{\text {par }}=1: 1.67 \times 10: 1.67 .
$$

It is concluded: various IEM influences, such as $d U_{\text {pol }}, d U_{d i a}$, and $d U_{\text {par, }}$ owing to EMF, are dominated compared to $d U_{S}$. In recognition that the flow had not been influenced by variation in temperature. In contrast, it was influenced by the EMF that was self-produced by the sudden motion of the rigid plate. 3D-Graphics illustrate the calculated variables' performances are shown, and their behavior is profoundly examined. Our finding concluded that: Our model and its solution and all calculated variables are compatible with IT laws.

\section{Nomenclature}

$\vec{B}$ : Induced magnetic field vector, $\boldsymbol{B}_{\mathbf{1}}$ : Induced magnetic field, $\overrightarrow{\boldsymbol{E}}$ : Induced electric induction vector, $\boldsymbol{E}$ : Induced electric field, $\overrightarrow{\boldsymbol{F}}$ : Lorentz's force vector, $\boldsymbol{f}$ : Electron velocity distribution function, $f_{0}$ : Equilibrium EVDF, $f_{1}$ : Frist component of EVDF, $f_{2}$ : Second component of EVDF, J: Current density, $J_{\mathbf{y}}^{(\mathbf{( s )}}$ : Entropy flux component, $\boldsymbol{K}_{\mathrm{B}}$ : Boltzmann constant $\left(\mathrm{Erg} / \mathrm{K}^{0}\right) 1.3810^{-16}, \boldsymbol{L}_{11}$ : Kinetic coefficient, $\boldsymbol{M}_{\mathrm{p}}$ : Plate Mach number, $\boldsymbol{M}$ : Particles Mach number, $\boldsymbol{P}$ : Polarization, $\boldsymbol{R}$ : Gas constant,
S: Entropy per unit mass, T: Temperature, $\boldsymbol{U}$ : Internal energy of the gas, $\boldsymbol{U}_{0}$ : Plate Velocity constant, $\boldsymbol{V}_{\mathbf{x}}$ : Mean velocity, $\boldsymbol{V}_{\mathbf{x} \mathbf{1}}$ : Mean velocity related to $f_{1}, \boldsymbol{V}_{\mathbf{x} \mathbf{2}}$ : Mean velocity related to $f_{2,} \boldsymbol{V}$ : Gas volume, $\boldsymbol{X}_{\mathrm{Mp}}$ : Thermodynamic force, $c_{0}$ : Speed of light, $\boldsymbol{C}$ : Particles' velocity, $\boldsymbol{d}$ : Particle's diameter, $\boldsymbol{e}$ : Electron's charge, $\boldsymbol{m}_{\mathrm{e}}$ : Electron's mass, $\boldsymbol{m}$ : Specific magnetization, $\boldsymbol{N}$ : Mean density, $\boldsymbol{n}_{\mathrm{e}}$ : Electron's concentration, $\boldsymbol{P}$ : Pressure, $\overrightarrow{\boldsymbol{r}}$ : Position vector of the particle, $\mathrm{t}$ : Time variable, $\overrightarrow{\boldsymbol{u}}$ : Mean velocity of the particle, $\boldsymbol{d} \boldsymbol{U}_{\mathrm{s}}:$ IEM due to entropy, $\boldsymbol{d} \boldsymbol{U}_{\mathrm{Pol}}$ : IEM due to polarization, $d U_{\text {par }}$ : IEM due to magnetization, $\boldsymbol{d} \boldsymbol{U}_{\text {dia }}$ : IEM due to magnetic field, $\boldsymbol{y}$ : Displacement variable, ': Dimensionless variable, e: Related to electrons, $\quad \boldsymbol{i}$ : Related to ions, $\boldsymbol{\tau}$ : Relaxation time, $\boldsymbol{\tau}_{x y}$ : Shear stress, $\boldsymbol{\mu}$ : Viscosity coefficient, $\boldsymbol{\lambda}$ : Mean free path, $\boldsymbol{\alpha}_{0}$ : Dimensionless parameter, $\boldsymbol{\alpha}$ : Damping constant.

\section{Abbreviations}

BGK: Bhatnagar-Gross-Krook, BE: Boltzmann kinetic equation, EMF: Electromagnetic Field, ES: Equilibrium State, IEM: Internal Energy Modification, MEMS: Micro-Electro-Mechanical Systems, NEMS: Nano-Electro-Mechanical Systems, EVDF: Electrons Velocity Distribution Function, RB: Rayleigh problem, RGP: Rarefied Gaseous Plasma.

\section{Data availability}

The data used to support the findings of this study are included in the article.

\section{Declaration of Competing Interest}

The authors declare that there is no conflict of interest regarding the publication of this paper.

\section{Acknowledgments}

This study is supported by the Egyptian Academy of Scientific Research and Technology by the associated grant number (No. 6508) under the ScienceUP Faculties of Science program.

Many thanks to the reviewers for their outstanding efforts in the review process. Many thanks to the editor-in-chief and all the journal editorial family members. 


\section{References}

1. Abdel Wahid, T. Z. (2012). Kinetic and thermodynamic treatment for the exact solution of the unsteady Rayleigh flow problem of a rarefied homogeneous charged gas. Journal of Non-Equilibrium Thermodynamics, 37(2): 119-141.

2. Michele, C. (2021). Lectures on the Mechanical Foundations of Thermodynamics. Springer Nature Switzerland AG.

3. Henry, C. F. (2022). Thermodynamics, Gas Dynamics, and Combustion. Springer Nature Switzerland AG.

4. Arastoopour, H., Gidaspow, D. and Lyczkowski, R. W. (2022). Transport Phenomena in Multiphase Systems. Springer Nature Switzerland AG.

5. Abourabia, A. M. and Abdel Wahid, T. Z. (2012). Kinetic and thermodynamic treatments of a neutral binary gas mixture affected by a non-linear thermal radiation field. Canadian Journal of Physics, 90(2): 137 149.

6. Abourabia, A. M. and Abdel Wahid, T. Z. (2011). Solution of the Krook kinetic equation model and non-equilibrium thermodynamics of a rarefied gas affected by a non-linear thermal radiation field. Journal of NonEquilibrium Thermodynamics, 36(1): $75-98$.

7. Shidloveskiy, V. P. (1967). Introduction to Dynamics of Rarefied Gases. Elsevier NY, pp.78 - 85.

8. Abourabia, A. M., Abdel Wahid, T. Z., Kinetic and thermodynamic treatment for the Rayleigh flow problem of an inhomogeneous charged gas mixture, Journal of NonEquilibrium Thermodynamics, 37(1), pp. 1-25, 2012.

9. Abourabia, A. M. and Abdel Wahid, T. Z. (2010). The unsteady Boltzmann kinetic equation and non-equilibrium thermodynamics of an electron gas for the Rayleigh flow problem. Canadian Journal of Physics, 88(7): 501 - 511.
10. El-Sakka, A. G., Abdellatif, R. A. and Montasser, S. A. (1985). Free Molecular Flow of Rarefied Gas Over an Oscillating Plate Under a Periodic External Force. Astrophysics and Distance Science, 109: 259 - 270.

11. Khater, A. H. and El-Sharif, A. E. (1988). Analytical solution of the Rayleigh's flow problem for a highly rarefied gas of a homogeneous system of plasma. Astrophysics and Distance Science, 146: 157 - 162.

12. Roshid, Md. M. and Roshid, H. (2018). Exact and explicit traveling wave solutions to two non-linear evolution equations which describe incompressible viscoelastic KelvinVoigt fluid. Heliyon, 4(8): 1 - 12.

13. Adel M Morad, S. M. A., Maize, A. A. and Nowaya, Y. S. R. (2021). A New Derivation of Exact Solutions for Incompressible Magnetohydrodynamic Plasma Turbulence. Journal of Nanofluids, 10(1): 98 - 105.

14. Abdel Wahid, T. Z. (2013). Travelling waves solution of the unsteady flow problem of a rarefied nonhomogeneous charged gas bounded by an oscillating plate, Mathematical Problems in Engineering, 113 .

15. Abdel Wahid, T.Z. (2013). Exact solution of the unsteady Krook kinetic model and nonequilibrium thermodynamic study for a rarefied gas affected by a non-linear thermal radiation field. Canadian Journal of Physics, 91(3): $201-210$

16. Abdel wahid, T. Z. and Elagan, S. K. (2012). Kinetic treatment for the exact solution of the unsteady Rayleigh flow problem of a rarefied homogeneous charged gas bounded by an oscillating plate. Canadian Journal of Physics, 90(10): 987 - 998.

17. Abdel Wahid, T. Z., Elsaid, E. M. and Morad, A. M. (2020). Exact solutions of plasma flow on a rigid oscillating plate under the effect of an external non-uniform electric field. Results in Physics, 19: 1 - 11. 
18. Abdel Wahid, T. Z. and Morad, A. M. (2020). On Analytical Solution of a Plasma Flow over a Moving Plate under the Effect of an Applied Magnetic Field. Advances in Mathematical Physics, 1 - 11.

19. Luz Muñoz-Ruiz, M., Parés, C. and Russo, G. (2021). Recent Advances in Numerical Methods for Hyperbolic PDE Systems. Springer Nature Switzerland AG.

20. Bhatnagar, P., Gross, E. and Krook, M. (1954). Model for collision processes in gases I. small amplitude processes in the charged and neutral one-component system. Phys. Rev.,94(3): 511-525.

21. Abdel Wahid, T. Z. (2020). On the irreversible thermodynamic of a gas influenced by a thermal radiation force generated from a heated rigid flat plate. Advances in Mechanical Engineering, 12(10): 1 - 21.

22. Abdel Wahid, T. Z. and Morad, A. M. (2020). Unsteady plasma flow near an oscillating rigid plane plate under the influence of an unsteady non-linear external magnetic field. IEEE Access, 8: 76423 - 76432.
23. Edward, M. P. (1965). Electricity and Magnetism. McGraw-Hill Book Co-Singapore 3rd.

24. Halid, B. and Igor, I. L. (2021). Nonequilibrium Thermodynamics, and Physical Kinetics. De Gruyter; 2nd edition.

25. Hatim, M. (2019). Extended NonEquilibrium Thermodynamics: From Principles to Applications in Nanosystems. CRC Press, 1st edition.

26. Baus, M. and Tejero, C. F. (2021). Equilibrium Statistical Physics. Springer Nature Switzerland AG.

27. Peter v. d. L. (1998). Thermodynamics Stability of Dia- And Paramagnetic Materials. Periodica Polytechnica Ser. Chem. Eng., 12(2): 97 -102.

28. Huba, J. D. (2019). NRL Plasma Formulary, Naval Research Laboratory, Washington DC, 20375.

29. Sitenko, A. G. (1967). Electromagnetic Fluctuation in Plasma. Academic Press, New York. 